\title{
Research Article \\ Effect of Hadron Contamination on Dielectron Signal Reconstruction in Heavy Flavor Production Measurements
}

\author{
Daniel Kikoła \\ Faculty of Physics, Warsaw University of Technology, Ulica Koszykowa 75, 00-662 Warsaw, Poland \\ Correspondence should be addressed to Daniel Kikoła; kikola@if.pw.edu.pl
}

Received 22 December 2014; Revised 20 February 2015; Accepted 22 February 2015

Academic Editor: Ming Liu

Copyright ( 2015 Daniel Kikoła. This is an open access article distributed under the Creative Commons Attribution License, which permits unrestricted use, distribution, and reproduction in any medium, provided the original work is properly cited. The publication of this article was funded by SCOAP S $^{3}$

\begin{abstract}
Dielectron signal reconstruction is an important tool for heavy flavor measurements because of its trigger feasibility and its relatively straightforward particle identification process. However, in the case of time projection chamber detectors, some hadron contamination is unavoidable, even if additional means are used to improve the particle identification process. In this paper, we investigate the effects of hadron (protons, pions, and kaons) contamination on the dielectron signal reconstruction process in the measurement of $J / \psi$ and electrons from heavy flavor hadron decays.
\end{abstract}

\section{Introduction}

The quark-gluon plasma (QGP) is a state of nuclear matter with properties that are determined by the quark and gluon degrees of freedom. This nuclear matter state existed in the early Universe, a few microseconds after the Big Bang. QGP can be created in the laboratory by collisions between heavy ions with relativistic energies $[1,2]$. The STAR and PHENIX experiments at the Relativistic Heavy Ion Collider (RHIC) at the Brookhaven National Laboratory and the ALICE experiment at the Large Hadron Collider at CERN are dedicated to the exploration of the properties of such a system.

We use an approach that is analogous to tomography to study the QGP in heavy ion collisions. An external penetrating probe, with properties (i.e., production mechanism) that are under experimental and theoretical control, propagates through the medium. We can then infer the properties of the system to be analyzed from the modification of the probe. Heavy quarks serve as suitable external-to-QGP probes. Because of their large masses, these quarks are produced very early in the collision in the initial interactions with large momentum transfer and before the QGP phase. Their production, in terms of both total and differential cross sections, is described well by perturbative quantum chromodynamics (QCD). Open heavy quark production is sensitive to the QGP dynamics and can be used to determine the fundamental properties of the QGP, such as its transport coefficients (see, e.g., $[3,4]$ and the references contained therein). Measurements of the production of various quarkonium states (the $J / \psi, \chi_{c}$, and $\Upsilon$ family) can provide insights into the thermodynamic properties of the QGP because their yields are expected to be modified in the hot nuclear matter, and different states would undergo modifications at different temperatures (see [5] and the references therein).

Open heavy quark production can be studied via the electrons from semileptonic decays of heavy flavor hadrons [6-9]. This is the most feasible approach found at RHIC to date, because the yields are large and a specialized high$p_{T}$ electron trigger can be used. However, this is a complex measurement process where a good understanding of all systematic effects is crucial [7] and especially of the background from the electrons produced by photon conversion in the detector and light meson decays. For studies of quarkonia, the dilepton channel is the most feasible experimental approach. The production of $b$ quarks can be also measured using $B \rightarrow$ $J / \psi$ via a displaced vertex reconstruction.

Electron identification and dielectron reconstruction are thus important aspects of heavy flavor measurements. The time projection chamber (TPC) is a tracking and particle 
identification detector that has been installed as part of the leading heavy ion experiments, including NA49/NA61, STAR, and ALICE. TPC uses the momentum dependences of specific ionizing energy losses for particle identification. However, there are momentum ranges where the $d E / d x$ bands for different species overlap and identification is thus hindered (e.g., electrons and protons for $p \sim 1 \mathrm{GeV} / c$ ). Both STAR and ALICE use additional means to improve particle identification (a time-of-flight detector at STAR and a time-of-flight and transition radiation detector at ALICE). However, it is difficult to have high purity in the overlapping range, even with ToF. For instance, hadron contamination in dielectron measurements of $\mathrm{Au}+\mathrm{Au}$ collisions at $\sqrt{s_{N N}}=$ $62.4 \mathrm{GeV}$ is $\sim 20 \%$ at $p \sim 0.7 \mathrm{GeV} / c$ (mostly because of misidentified kaons) and $\sim 40 \%$ at $p \sim 1.1 \mathrm{GeV} / c[10]$ (mostly because of pion contamination), even with ToF in place.

Hadron misidentification also affects the heavy flavor correlation measurements $\left(e^{H F}-e^{H F}\right.$ or charmed meson $-e^{H F}$ correlations) that provide additional insights into heavy quark interactions with nuclear matter. It is important to distinguish these correlations from light meson correlations (charged hadrons and $\pi^{0}$ ), which probe the interactions of gluons and light quarks with nuclear matter. When the hadron contamination is high, the $e^{H F}-e^{H F}$ correlations are dominated by light flavor jet correlations. If the photonic background is underestimated, then electrons from the $\pi^{0}$ and $\eta$ Dalitz decays and $\gamma$ conversion will contaminate the results with light flavor jet correlations. When the photonic background is overestimated, then the observed correlations will be distorted because the background correlations will be oversubtracted. It is essential to have the hadron contamination well under control in such measurements.

In this paper, we investigate the effects of hadron contamination on heavy flavor measurements with a TPC. We focus on two experimental techniques that involve dielectron signal reconstruction: measurement of $J / \psi$ production and the so-called photonic background estimation in the heavy flavor electron measurements at STAR and ALICE. The goal of this study is to estimate any possible biases or systematic effects due to hadron misidentification, that is, to study how a combinatorial background (and thus the statistical uncertainty) changes and whether the measured yield is biased because of the correlated background introduced by hadron contamination. We begin with a brief description of the simulation setup and of how the hadron contamination is modeled. Then, we present the results that were obtained for different hadron contamination levels.

\section{Simulation Setup}

We used the PYTHIA 6.4 [11] event generator to generate 1 billion minimum-bias $p+p$ events at $\sqrt{s}=200 \mathrm{GeV}$. Most of the PYTHIA settings were at their default values, apart from the following parameters: the charm quark mass $m_{c}=1.25 \mathrm{GeV} / c^{2}(\operatorname{PMAS}(4,1)=1.25)$, the bottom quark mass $m_{b}=4.1 \mathrm{GeV} / c^{2}(\operatorname{PMAS}(5,1)=4.1), \operatorname{MSTP}(32)=4$ $\left(Q^{2}\right.$ scale), the width of the primordial parton traverse momentum distribution in the hadron $\left\langle k_{T}\right\rangle=$
$1.5 \mathrm{GeV} / c(\operatorname{PARP}(91)=1.5)$, and the $K$ factor for hard cross sections for parton-parton interactions $K=3.5(\mathrm{PARP}(31)=$ 3.5). These parameters were modified based on the work of [12] to model a correlated background from the $c \bar{c}$ and $b \bar{b}$ continuum in the $J / \psi$ signal studies.

Here we consider an idealized detector with acceptance and particle identification that are similar to the STAR TPC [13]. We select charged particles with $p_{T}>0.2 \mathrm{GeV} / c$ and $|y|<1$. The transverse momentum resolution is parametrized as a Gaussian with a standard deviation $\sigma\left(p_{T}\right)$ that is given by $\left(\sigma\left(p_{T}\right) / p_{T}\right)^{2}=a^{2}+\left(b p_{T}\right)^{2}$, where $a=0.005$ and $b=0.00012$. This functional form is chosen based on the $p_{T}$ resolution for pions in the ALICE TPC [14], and the $a$ and $b$ parameters were selected to match the mass resolution of the $J / \psi$ signal in the data.

$J / \psi$ production is simulated using the TPythia8Decayer class from the ROOT framework [15] with the $J / \psi p_{T}$ spectrum at RHIC at midrapidity [16] used as an input. In total, $1650 \mathrm{~J} / \psi$ 's were generated. The radiative decays of $J / \psi\left(J / \psi \rightarrow e^{+} e^{-} \gamma, B R=(8.8 \pm 1.4) \times 10^{-3}\right)\left(\right.$ for $E_{\gamma}>$ $100 \mathrm{MeV}$ ) [17] affect this study because the electrons from this process have lower momentum. The observed radiative decays (also known as internal radiation) amount to $15 \%$ of the $J / \psi \rightarrow e^{+} e^{-}$decays $(B R=5.94 \pm 0.06 \%)$ [17], which leads to a low mass tail visible in measured mass distribution shown in Figure 1 for $2.6<M_{e e}<3 \mathrm{GeV} / c^{2}$. We modeled the $e^{+} e^{-}$mass distribution from a radiative decay using the power-law form $f\left(m_{e e}\right)=\left[n /\left|\alpha_{\text {rad }}\right|-\left|\alpha_{\text {rad }}\right|-m_{e e}+m_{J / \psi}\right]^{-n_{\text {rad }}}$, where $2<m_{e e}<3.04 \mathrm{GeV} / c^{2}, m_{J / \psi}=3.0969 \mathrm{GeV} / c^{2}$ is a $J / \psi$ mass, $\alpha_{\text {rad }}=1.18$, and $n_{\text {rad }}=1.45$. This modified mass spectrum is passed to TPythia8Decayer to account for the radiative decays. We tuned the parameters of a mass distribution and a $p_{T}$ resolution to match the $J / \psi$ mass spectrum reported by STAR in $p+p$ collisions [18] for $2<$ $p_{T}<6 \mathrm{GeV} / c$ (Figure 1(a)). There is reasonable agreement between the simulations and the data for the chosen values of $a, b, \alpha_{\text {rad }}$, and $n_{\text {rad }}$.

For yield extraction in Section 3, we parametrize the $J / \psi$ signal with a Crystal Ball function $[19,20]$. This function consists of a Gaussian core (which models the mass resolution) and a power-law low-end tail for the energy loss processes:

$$
\begin{aligned}
& f(x, \alpha, \bar{x}, n, \sigma) \\
& =N \begin{cases}\exp \left(\frac{(x-\bar{x})^{2}}{2 \sigma^{2}}\right) & \text { if } \frac{(x-\bar{x})}{\sigma}>-\alpha ; \\
A\left(B-\frac{(x-\bar{x})}{\sigma}\right)^{-n} & \text { if } \frac{(x-\bar{x})}{\sigma} \leq-\alpha,\end{cases}
\end{aligned}
$$

where

(i) $N$ is a normalization factor;

(ii) $\bar{x}$ is the mean value of the core Gaussian function; $\bar{x}$ accounts for a possible offset from the nominal of $J / \psi$ mass value;

(iii) $\sigma$ is the mass resolution;

(iv) $\alpha$ and $n$ are parameters that describe the power law tail; $\alpha$ defines the joining point and $n$ is the power that characterizes the strength of the energy loss process; 


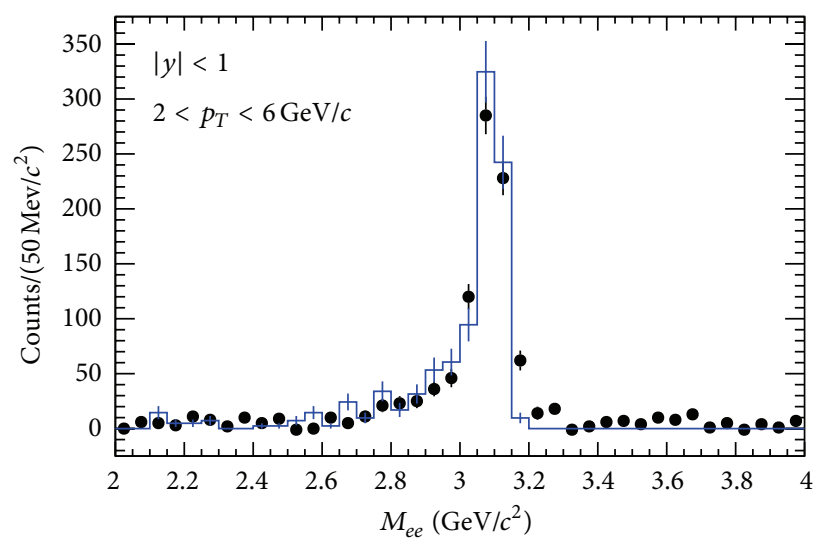

- $\operatorname{STAR} p+p$ Simulations

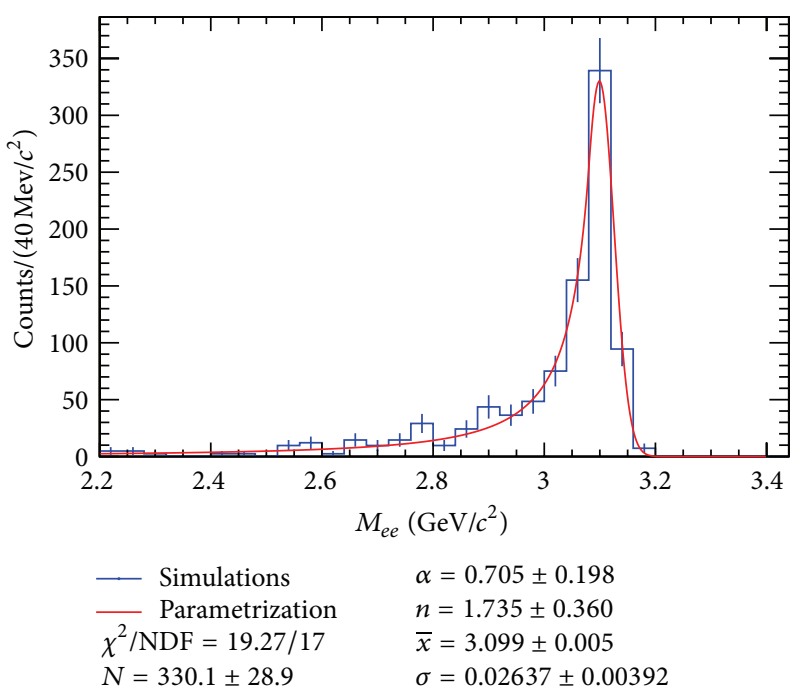

(b)

Figure 1: (a) $J / \psi$ signal in $p+p$ collisions at $200 \mathrm{GeV}$ compared to the simulations. (b) Simulated $J / \psi$ signal compared to a Crystal Ball function fitting used to account for the contribution of $J / \psi \rightarrow e^{+} e^{-} \gamma$.

(v) $A=(n /|\alpha|)^{n} \exp \left(-|\alpha|^{2} / 2\right)$ and $B=n /|\alpha|-|\alpha|$ are terms that are determined from the requirement that the function itself and its first derivative are continuous.

The parameters $N, \alpha, \sigma, n$, and $\bar{x}$ in function 1 are obtained from a fitting to the simulated mass distribution shown in Figure 1(b). This model matches the generated data reasonably well $\left(\chi^{2} / \mathrm{NDF}=19 / 17\right)$. The Crystal Ball function is not used directly for signal extraction. We use this function together with residual background parametrization in the fitting to the mass spectrum to fix the parameters for the residual background; the fitting procedure is described in Section 3.

For hadron contamination, we study the misidentification of kaons, protons, and pions as electrons with particular focus on kaon and proton contamination. The pion and electron $d E / d x$ bands begin to overlap at high levels of momentum and the pion contamination is assumed to be negligible for $p_{T}<1.25 \mathrm{GeV} / c$. Based on the $d E / d x$ dependence as a function of momentum in both STAR and ALICE $[13,21]$, we assumed that kaons with momentum of $0.45<p<0.55 \mathrm{GeV} / c$ and protons with momentum of $0.85<p<1.05 \mathrm{GeV} / c$ could be misidentified as electrons. At higher levels of transverse momentum, pions are the dominant source of the hadron contamination and, for $p_{T}>$ $1.25 \mathrm{GeV} / c$, we use the parametrizations shown in Figure 2 to simulate that effect. We began with the electron purity (the fraction of electrons in a particle sample) in $p+p$ collisions at $\sqrt{s}=200 \sim \mathrm{GeV}$ reported in [6] and fitted the data using a Gaussian function to obtain a default parametrization of the electron purity (parametrization 1). To vary the level of pion contamination, we simply modify parametrization 1: we maintain the same amplitude and mean value but vary the width as follows:

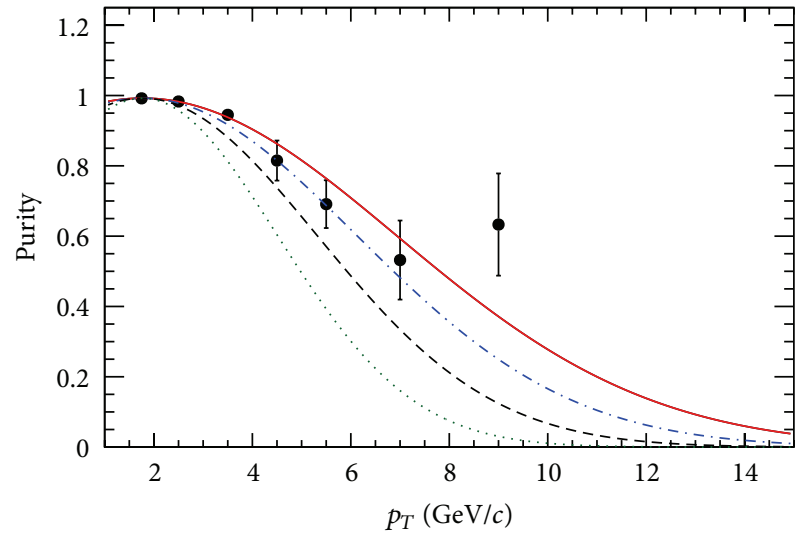

$\begin{array}{lll}\text { - } & \text { STAR } p+p 200 \mathrm{GeV} & --- \text { Parametrization } 3 \\ \text { - Parametrization } 1 & \ldots . . & \text { Parametrization } 4 \\ \text { - - Parametrization } 2 & & \end{array}$

FIGURE 2: Electron purity measured by STAR in $p+p$ collisions at $\sqrt{s}=200 \mathrm{GeV}[6]$. The data are fitted using a Gaussian function ("parametrization 1"). We obtained parametrizations 2 to 4 by changing the width of parametrization 1 by 1,2 , and 3 uncertainties of the width of parametrization 1.

(i) parametrization 2: $\sigma_{2}=\sigma_{1}-\delta\left(\sigma_{1}\right)$, where $\sigma_{1}$ is the width of the Gaussian function from the fitting to the STAR data and $\delta\left(\sigma_{1}\right)$ is its uncertainty;

(ii) parametrization 3: $\sigma_{3}=\sigma_{1}-2 \delta\left(\sigma_{1}\right)$;

(iii) parametrization 4: $\sigma_{4}=\sigma_{1}-3 \delta\left(\sigma_{1}\right)$.

Figure 2 shows these parametrizations.

We consider five possible scenarios for particle identification. 


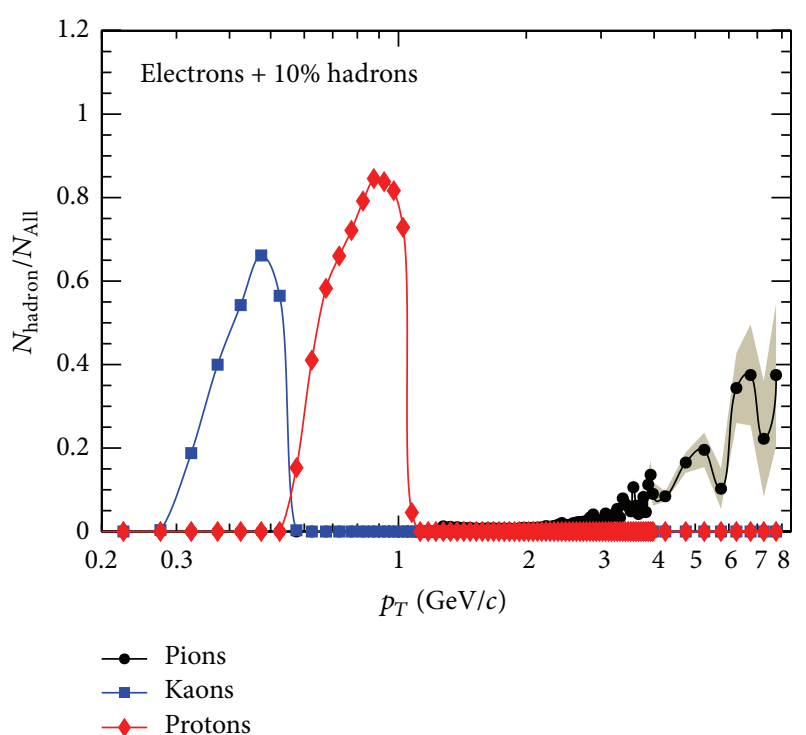

(a)

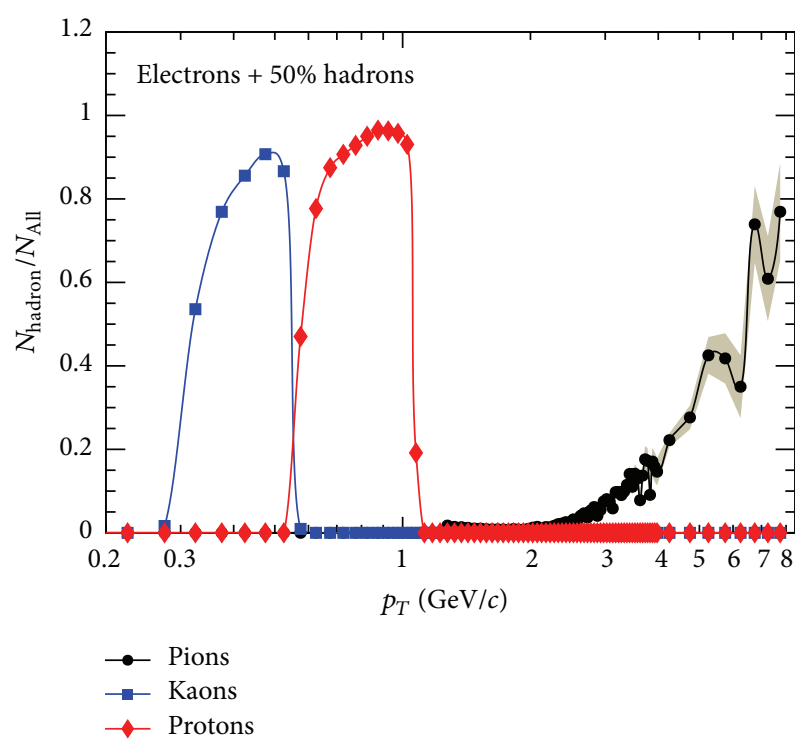

(c)

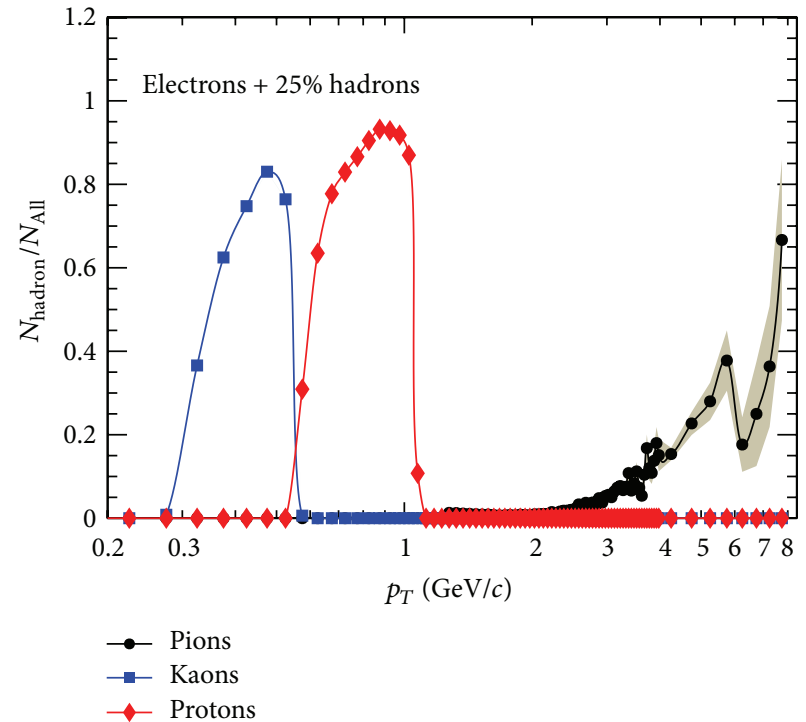

(b)

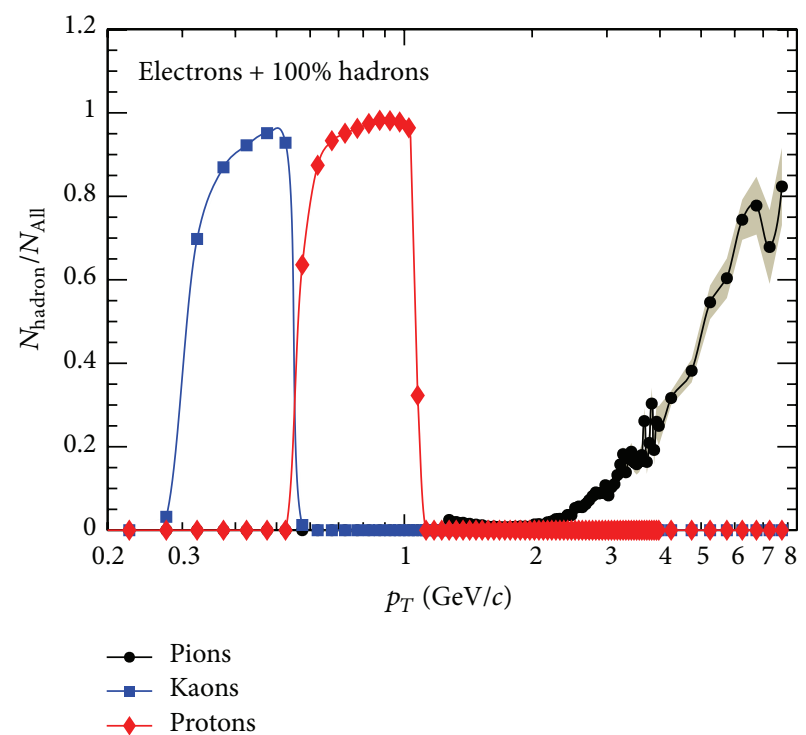

(d)

FIGURE 3: Relative hadron contributions to inclusive samples in the analysis for different hadron misidentification levels.

(i) "Electrons only": idealized particle identification, where all electrons are selected and all hadrons are rejected.

(ii) "Electrons $+10 \%$ hadrons": all electrons are selected for the analysis, plus $10 \%$ of the kaons and protons in the crossover ranges are misidentified as electrons, and the purity at high $p_{T}$ after pion contamination is given by parametrization 1 in Figure 2.

(iii) "Electrons $+25 \%$ hadrons": the same as the above, but $25 \%$ of the hadrons in the crossover ranges are misidentified as electrons in this case, and the purity at high $p_{T}$ is given by parametrization 2 in Figure 2 .

(iv) "Electrons $+50 \%$ hadrons": the same as the above, but $50 \%$ of the hadrons in the crossover ranges are misidentified as electrons in this case, and the purity at high $p_{T}$ is given by parametrization 3 in Figure 2.

(v) "Electrons $+100 \%$ hadrons": the same as the above, but all hadrons in the crossover ranges are misidentified as electrons in this case, and the purity at high $p_{T}$ is given by parametrization 4 in Figure 2 .

We select hadrons by randomly sampling the tracks in the overlap range until a given level of misidentification is met. Figure 4 shows the purity of the tracks for the studied misidentification levels. The purity here is defined as the fraction of electrons relative to the total number of particles selected for the invariant mass reconstruction according to the particle identification scenarios listed above. Even for the lowest misidentification level considered, the purity at 


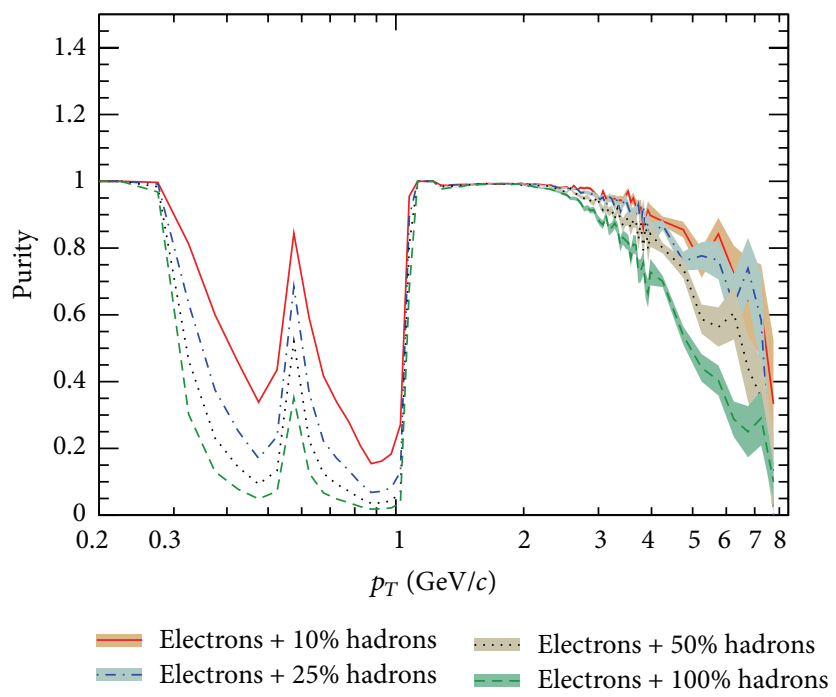

FIgURE 4: Purity, defined as the fraction of electrons in each particle sample in the analysis, for different hadron misidentification levels.

TABLE 1: Signal in the $J / \psi$ mass range of $2.7<m_{e^{+} e^{-}}<3.3 \mathrm{GeV} / c^{2}$ for various hadron contamination levels.

\begin{tabular}{lcccc}
\hline & Signal & $S / B$ ratio & Significance & Goodness of fit: $\chi^{2} / \mathrm{NDF}$ \\
\hline Electrons only & $1525 \pm 50$ & 4.3 & 32 & $46.9 / 33$ \\
Ele. $+10 \%$ hadr. & $1500 \pm 53$ & 2.9 & 30 & $38.7 / 33$ \\
Ele. $+25 \%$ hadr. & $1477 \pm 58$ & 1.9 & 27 & $41.3 / 33$ \\
Ele. $+50 \%$ hadr. & $1513 \pm 64$ & 1.3 & 24 & $33.2 / 33$ \\
Ele. $+100 \%$ hadr. & $1424 \pm 76$ & 0.7 & 19 & $49.8 / 33$ \\
\hline
\end{tabular}

low $p_{T}$ in the overlap ranges is low at $40 \%$ for the electronkaon overlap and $20 \%$ for the electron-proton overlap, which are both lower than previously obtained experimental values [22]. The "Electrons $+10 \%$ hadrons" scenario thus already provides an upper limit on the effects that could be observed experimentally. We use the higher misidentification levels as case studies for purity versus signal significance interplay.

Each particle is assumed to have the mass of an electron and each particle is paired with opposite-sign partners to form an invariant mass spectrum. The combinatorial background is estimated using the sum of all like-sign pairs from the same event. Figure 5 shows a mass spectrum for the foreground (all opposite-sign pairs) and the combinatorial background for the integrated $p_{T}$ for the selected levels of hadron misidentification: (a) "Electrons only," (b) "Electrons + 25\% hadrons," (c) "Electrons + 50\% hadrons," and (d) "Electrons $+100 \%$ hadrons." The resonance peaks for $\pi^{0}, \eta, \rho$, $\omega$, and $J / \psi$ are all clearly visible in the case of the pure electron sample in Figure 5(a). When the hadron contamination increases, the combinatorial background in the mass spectra shown in Figures 5(b)-5(d) increases and its shape changes. A correlated background due to the hadron misidentification arises with increasing contamination level, with a notable $\phi \rightarrow K^{+} K^{-}$peak around $m_{e e} \sim 0.26 \mathrm{GeV} / c^{2}$, which is reconstructed at a lower mass because a wrong daughter mass assumption was made in the invariant mass calculation.

Figure 6 shows an example of a correlated background induced by hadrons that has been broken down into its sources (various hadron-hadron and hadron-electron pairs) (Figure 6(a)) and is then shown as a cumulative distribution (Figure 6(b)). Kaon-kaon and proton-proton pairs are the two largest background sources, which are produced by $\phi \rightarrow K^{+} K^{-}$decays and correlated signals from jets. The sharp cut-offs for $K-K$ and $p-p$ pairs (1.1 and $2.1 \mathrm{GeV} / c^{2}$ ) occur because of the momentum ranges that are assumed for kaon and proton contamination $\left(p^{\text {proton }}<\right.$ $\left.1.05 \mathrm{GeV} / c, p^{\text {kaon }}<0.55 \mathrm{GeV} / c\right)$. Any pair mass that is reconstructed under the electron mass assumption then has a maximum value of $1.1 \mathrm{GeV} / c^{2}$ or $2.1 \mathrm{GeV} / c^{2}$ for the $K-K$ and $p-p$ pairs, respectively. Pion contamination does not have a noticeable impact on the $p_{T}$-integrated signal because the pion contamination is very low for $p_{T}<2 \mathrm{GeV} / c$ (Figure 3 ) and the high- $p_{T}$ pion yield is small when compared with that of the low- $p_{T}$ hadrons.

In the next two sections, we focus on two parts of the dielectron spectrum: the $J / \psi$ mass range and $m_{e e}<$ $0.2 \mathrm{GeV} / c^{2}$, which is used to estimate the photonic background in heavy flavor electron analysis $[6,7,9]$.

\section{3. $J / \psi$ Signal}

Figures $7(\mathrm{a}), 7(\mathrm{c}), 7(\mathrm{e}), 7(\mathrm{~g})$, and $7(\mathrm{i})$ show invariant mass spectra in a $J / \psi$ mass range for a foreground (all $e^{+} e^{-}$pairs, $N_{+-}$) and a combinatorial background (sum of like-sign pairs, $N_{++}+N_{--}$) for the different misidentification levels. 


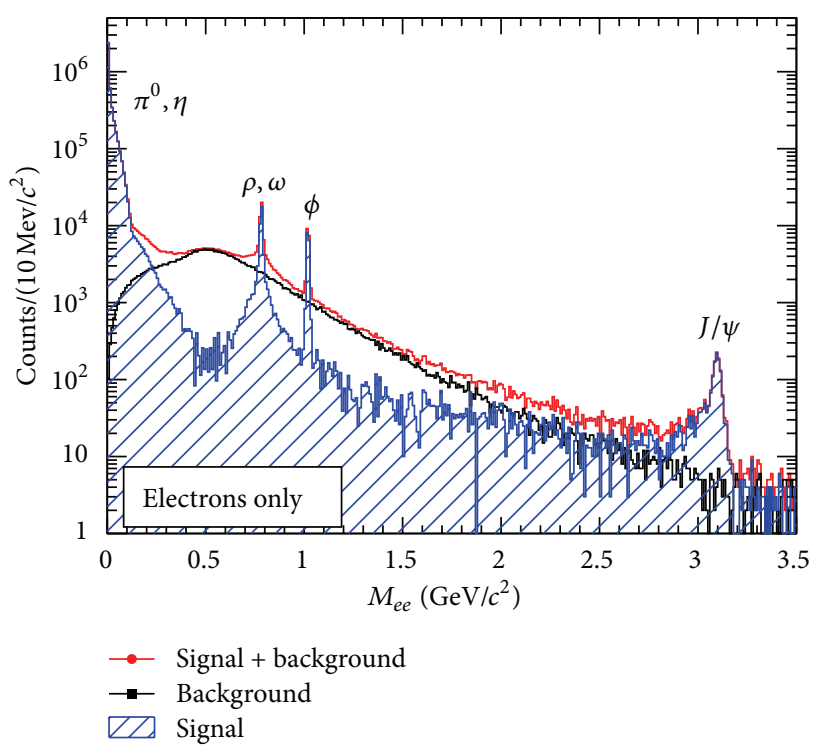

(a)

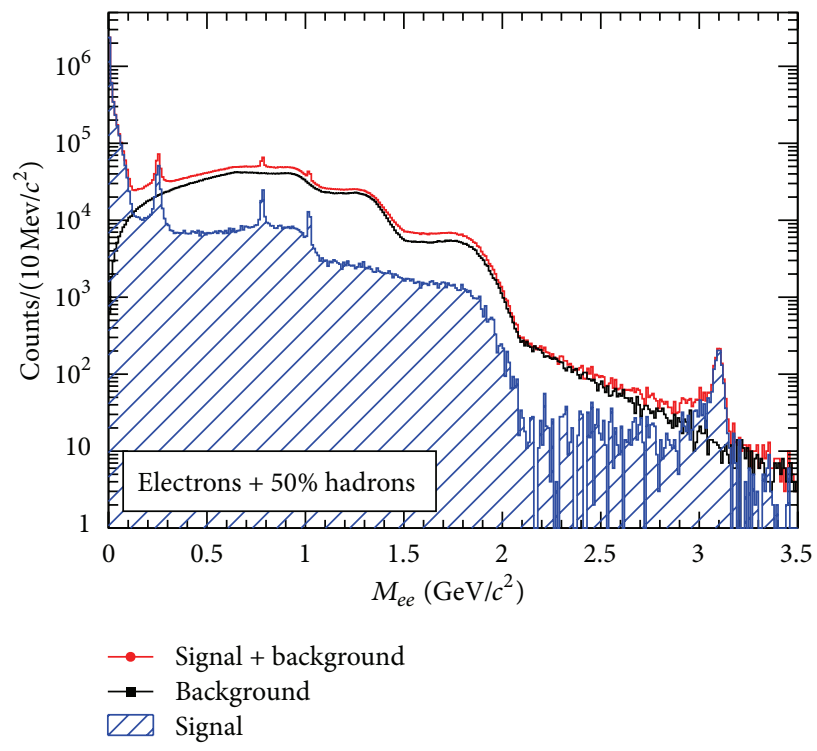

(c)

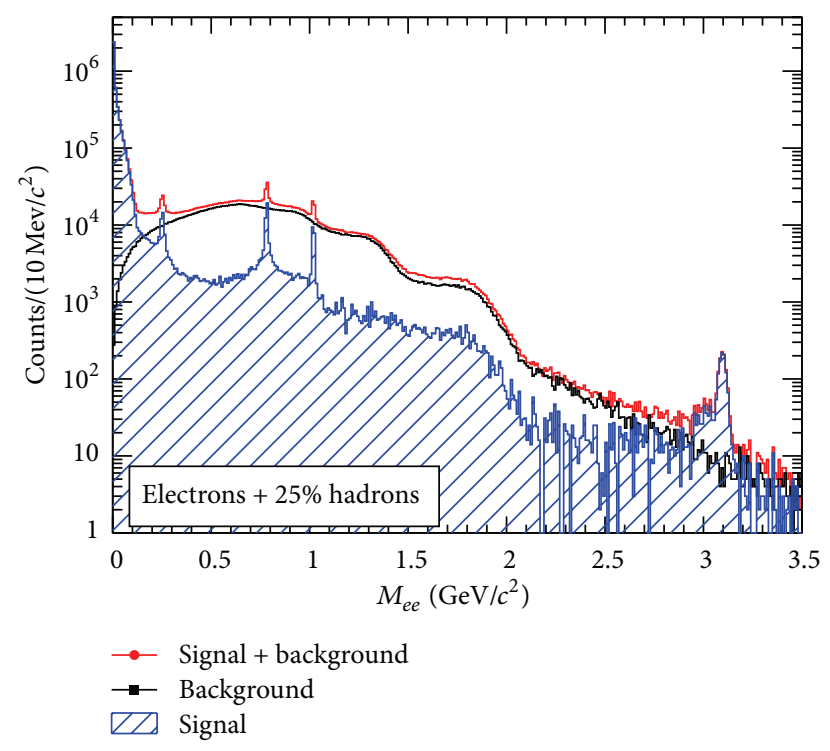

(b)

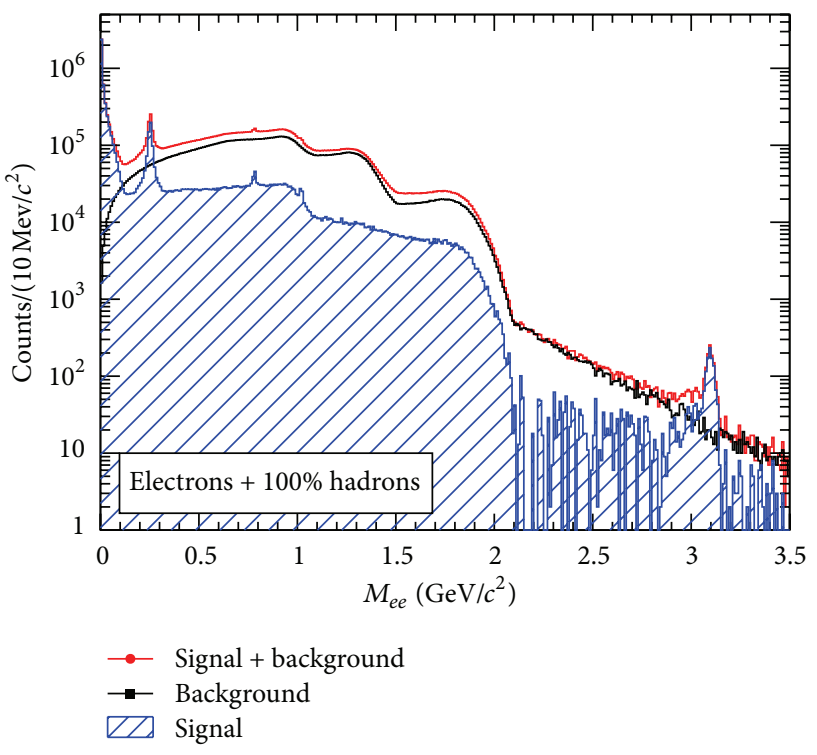

(d)

FIGURE 5: Invariant mass spectrum for foreground (all opposite-sign pairs) and combinatorial background for selected levels of hadron misidentification: (a) "Electrons only," (b) "Electrons + 25\% hadrons," (c) "Electrons + 50\% hadrons," and (d) "Electrons + 100\% hadrons."

The dielectron signals after subtraction of the combinatorial backgrounds are shown in Figures 7(b), 7(d), 7(f), 7(h), and $7(\mathrm{j})$. We fit the dielectron signals using a sum of the Crystal Ball function (function 1) for the $J / \psi$ signal (which also includes $J / \psi \rightarrow e^{+} e^{-} \gamma$ ) and an exponential function (for the residual background from the charm continuum). The Crystal Ball parameters $\alpha, \sigma, n$, and $\bar{x}$ are fixed based on the fitting to the mass distribution shown in Figure 1(b). The normalization $N$ and the parameters of the exponential function are free in the fitting process.

The fit is performed using the ROOT framework in a mass range of $2.2<m_{e e}<4 \mathrm{GeV} / c^{2}$ using the $\chi^{2}$ method, and the results are shown in Figure 7 and in Table 1.
The band in Figure 7 represents a $1 \sigma$ uncertainty contour from the fitting. The signal in Table 1 is obtained by counting the entries in the mass range of $2.7<m_{\mathrm{e}^{+} \mathrm{e}^{-}}<3.3 \mathrm{GeV} / c^{2}$ and then subtracting the residual background. We calculate the uncertainty based on the residual background, $\sigma_{R G}$, while taking the correlations of the fitting parameters into account. We then propagate $\sigma_{R G}$ to the final $J / \psi$ uncertainty by assuming that these two uncertainties are uncorrelated.

The combinatorial background increases with increasing hadron contamination, and thus the signal-to-background ratio decreases while the statistical uncertainty increases. For the highest hadron contamination, the background is larger by a factor of six, and the uncertainty increases by $50 \%$ when 


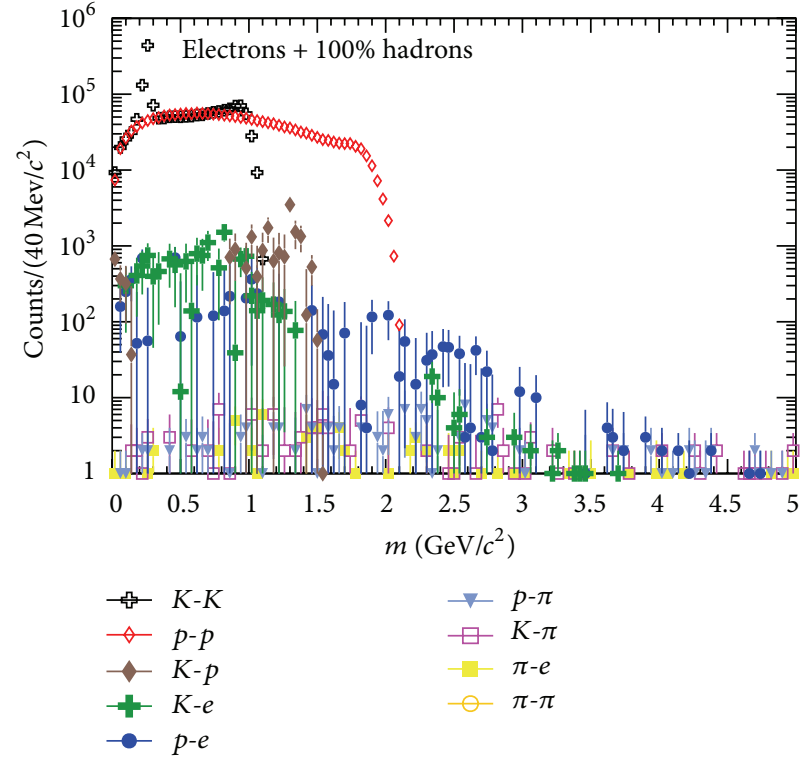

(a)

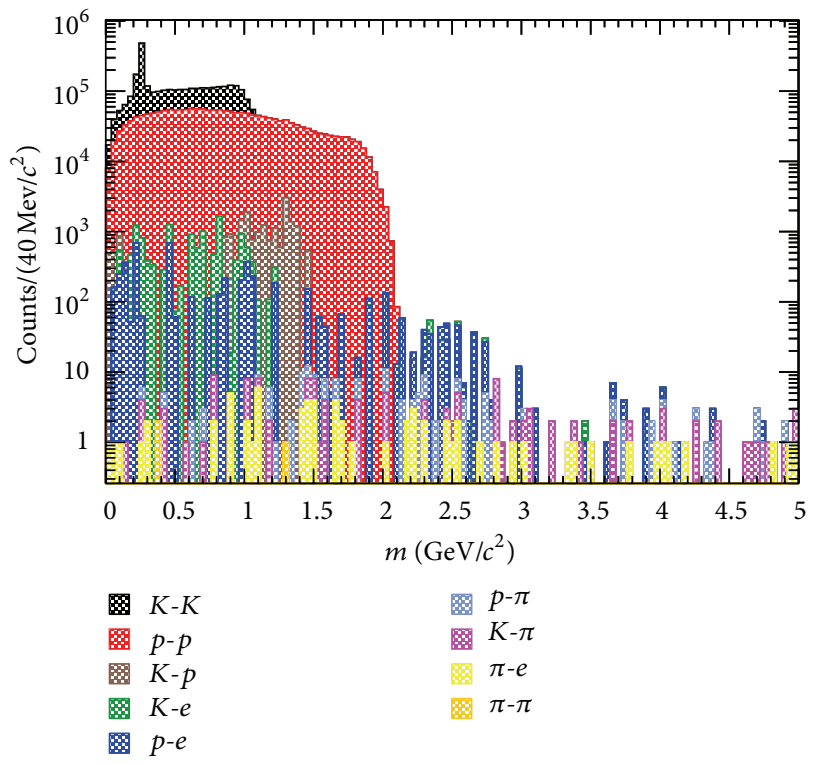

(b)

FIGURE 6: Correlated background induced by hadrons shown as (a) a mass distribution for each pair and (b) a cumulative (stacked) distribution broken down into its constituent components.

compared with a pure electron sample. The low mass tail due to $J / \psi \rightarrow e^{+} e^{-} \gamma$ is visible for the pure electrons and for hadron contamination of less than $50 \%$. When hadron misidentification increases and thus the combinatorial background increases, then the radiative decay tail in $2.6<m_{e e}<$ $2.8 \mathrm{GeV} / c^{2}$ is hidden by the fluctuations and is difficult to recover from the data. This effect is present in general when the signal-to-background ratio is low, for example, in $[23,24]$. Overall, the extracted $J / \psi$ signal is consistent within the statistical uncertainty with the simulated yield $(1500 \mathrm{~J} / \psi$ 's in $2.7<m_{e^{+} e^{-}}<3.3 \mathrm{GeV} / c^{2}$ ). At the highest contamination level, the extracted yield is $5 \%$ lower than the real value. However, this difference is not statistically significant.

\section{Photonic Electron Signal}

Heavy quark production can be studied via the electrons from the semileptonic decays of heavy flavor hadrons $\left(e^{H F}\right)[6-$ 8]. However, it requires complex measurements, whereby a good understanding of all the systematic effects is crucial [7]. The main background in these analyses comes from the so-called photonic electrons, that is, electrons from photon conversions in the detector material and from Dalitz decays of the $\pi^{0}$ and $\eta$ mesons $\left(\pi^{0} \rightarrow \gamma e^{+} e^{-}\right.$and $\left.\eta \rightarrow \gamma e^{+} e^{-}\right)$. One possible strategy is to identify the photonic background using a statistical approach as a signal in the low mass region of the dielectron $m_{e^{+} e^{-}}$mass spectrum (e.g., $m_{e^{+} e^{-}}<$ $\left.0.15 \mathrm{GeV} / \mathrm{c}^{2}\right)[6,9]$. Each primary photonic electron candidate is paired with an opposite-sign electron in an event, and the combinatorial background is estimated using like-sign pairs. The photonic electron yield is calculated by $N_{\text {pho }}=$ $\left(N^{U L}-N^{L S}\right) / \epsilon_{\text {pho }}$, where $N^{U L}$ and $N^{L S}$ are the numbers of the opposite-sign and like-sign pairs, respectively. $\epsilon_{\text {pho }}$ is the photonic electron reconstruction efficiency, which is determined from full GEANT simulations of the detector. $\epsilon_{\text {pho }}$ is a function of $p_{T}$ and varies from $15 \%$ at $0.5 \mathrm{GeV} / c$ to $60 \%$ at $7 \mathrm{GeV} / c$ [22]. The photonic electrons are also used to obtain high-purity electron samples to calculate the electron identification efficiency [23]. In other analyses, the background is determined via a cocktail simulation [8, 25]. In that approach, an inclusive electron spectrum is first measured, then the electrons from various background sources are simulated using a Monte Carlo hadron-decay generator, and finally these electrons are subtracted from the inclusive electron spectra. This method requires a good knowledge of the input momentum spectra of the potential background sources. Here we focus on the first, statistical approach.

We identify the photonic electron as a particle that has a partner in the TPC $\left(p_{T}>0.2 \mathrm{GeV} / c,|\eta|<1\right)$ and the invariant mass of this pair is $m_{e^{+}} e^{-}<0.2 \mathrm{GeV} / c^{2}$. The photonic signal is shown in Figure 8 and in Table 2. Based on the statistics used in this study, an increase in the statistical uncertainty because of a larger background is negligible. The integrated $p_{T}$ signal has a weak dependence on the hadron contamination level. The bias, which is defined as a relative difference compared to the signal extracted from the pure electron sample, is less than $2 \%$ for maximum hadron contamination of less than 50\% (Figure 8 and Table 2).

However, the bias depends strongly on the electron $p_{T}$. We extracted the photonic signal as a function of the single electron $p_{T}$ and then compared it with the signal extracted from the pure electron sample. To quantify the bias, we calculated the relative difference in the extracted photonic signal yield when compared with the pure electron case, 


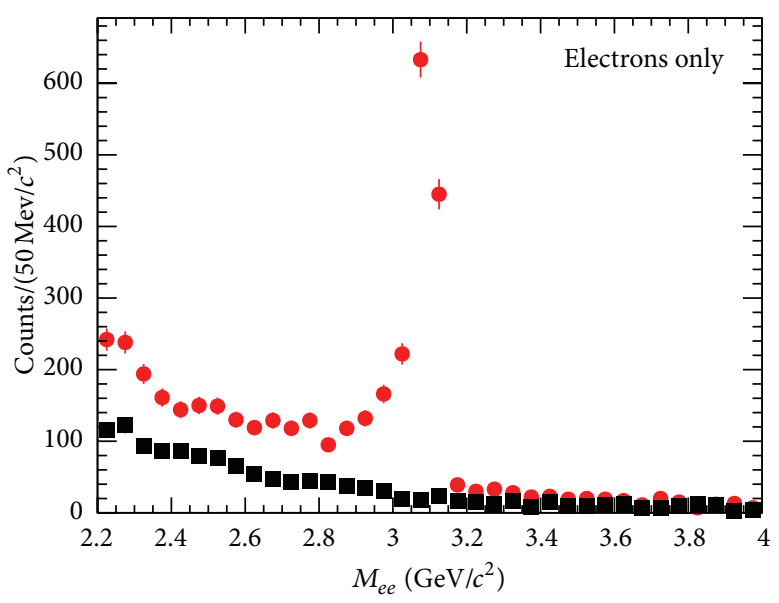

- $\mathrm{N}_{+-}$

- $N_{++}^{+-}+N_{--}$

(a)

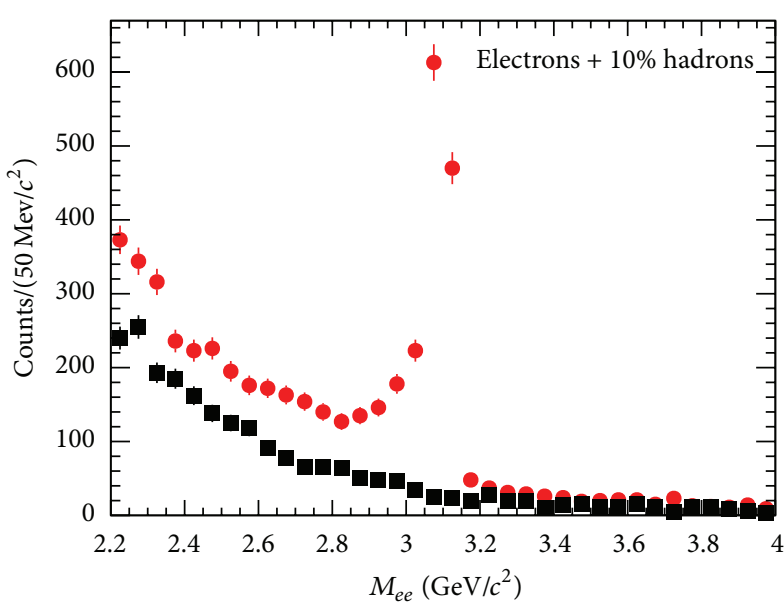

$N_{+-}$
- $N_{++}+N_{--}$

(c)

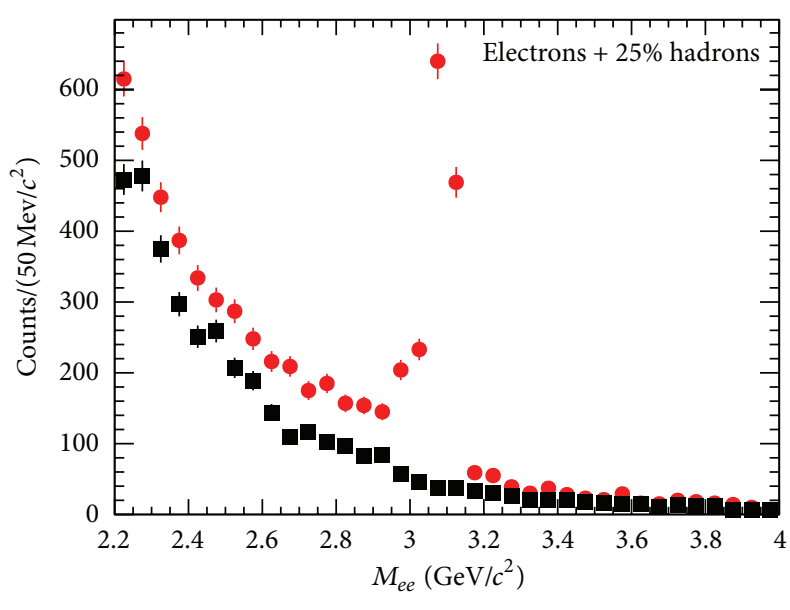

- $\mathrm{N}_{+-}$

- $N_{++}^{+-}+N_{--}$

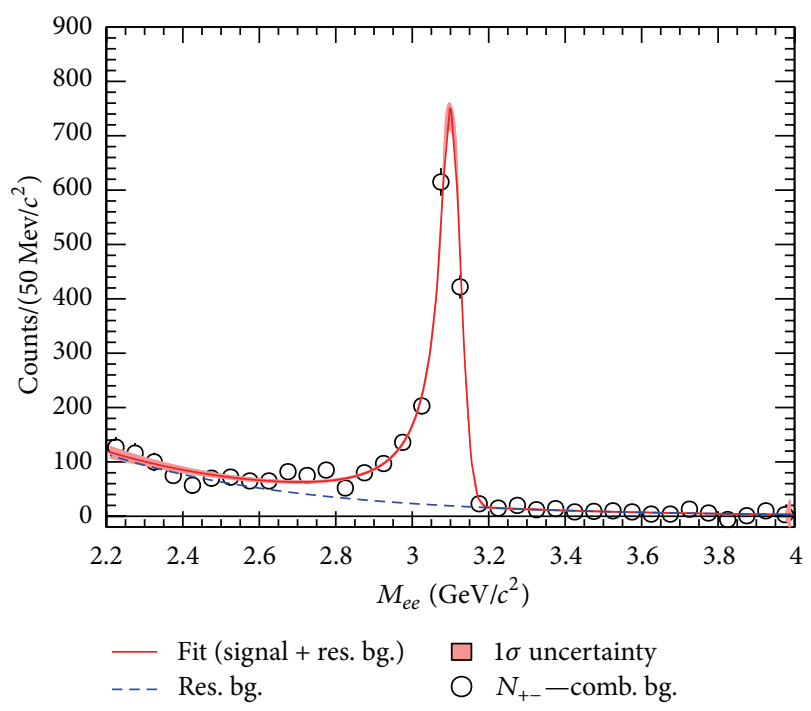

(b)

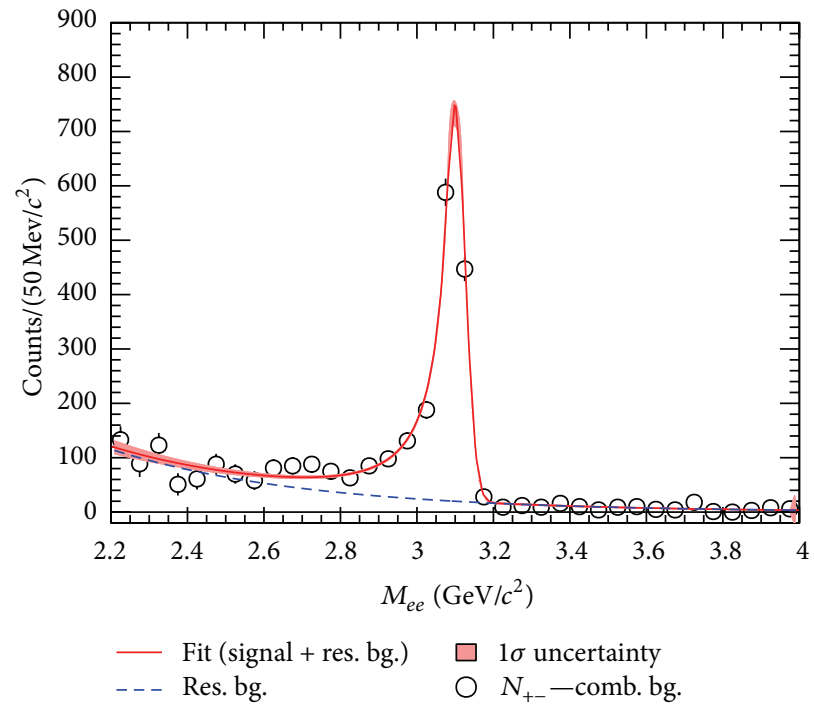

(d)

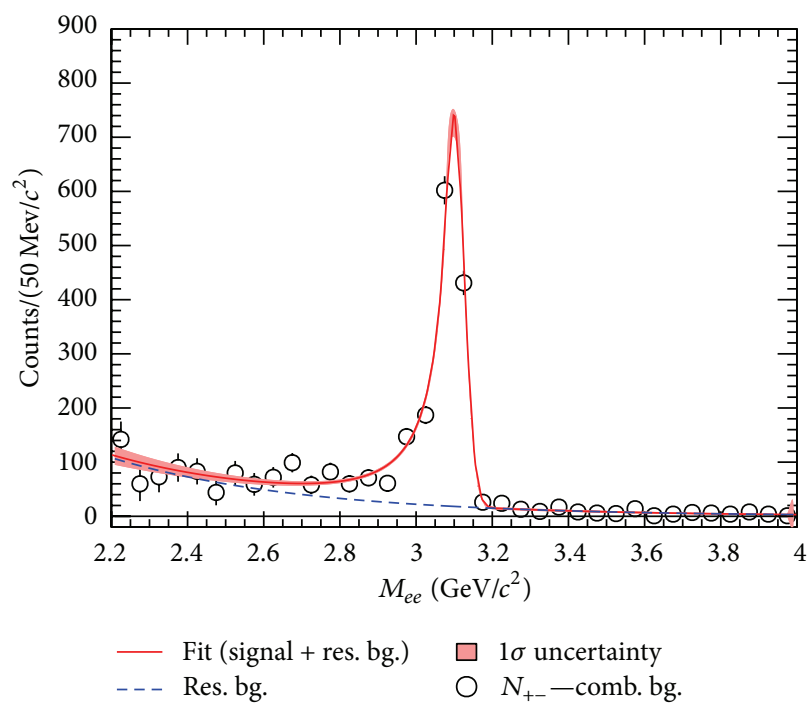

(f)

Figure 7: Continued. 


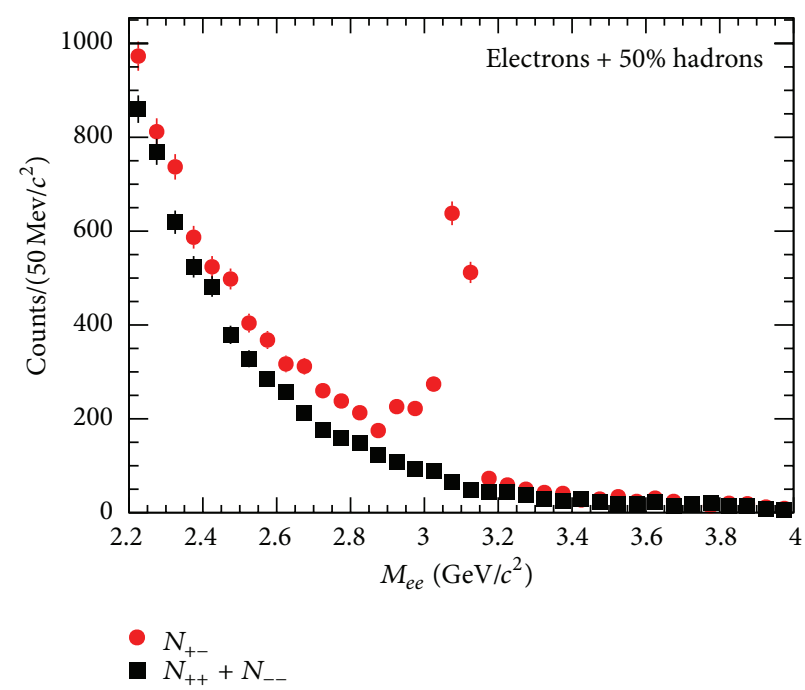

(g)

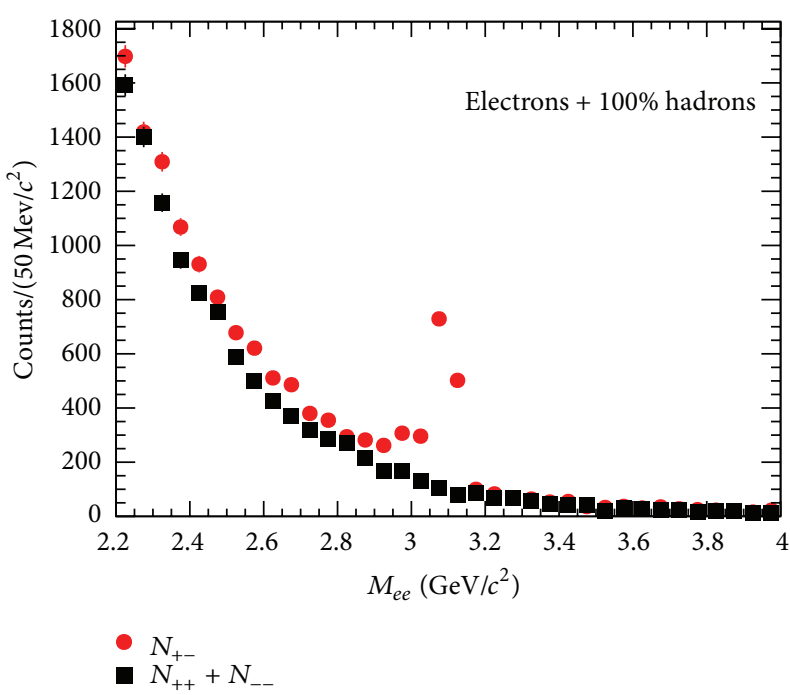

(i)

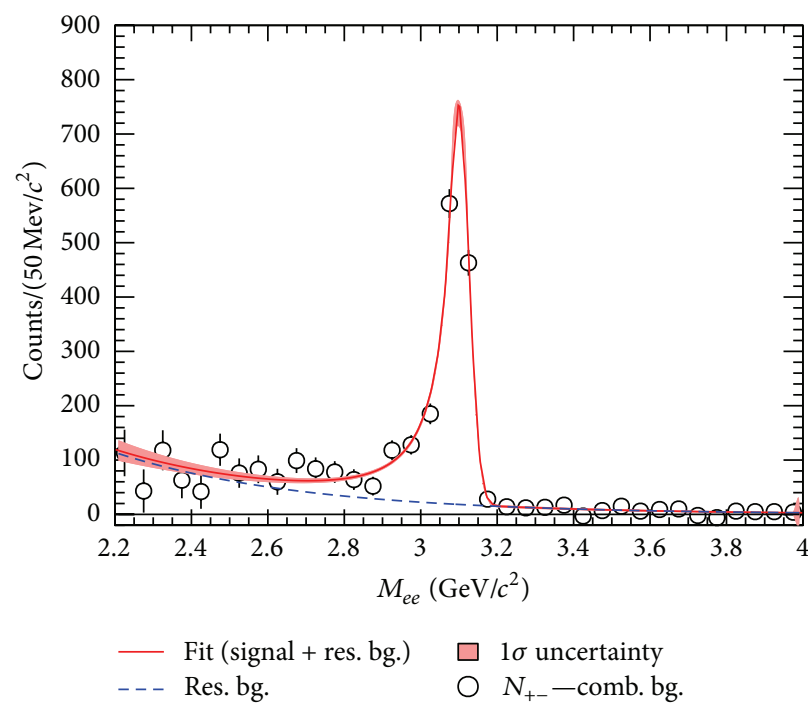

(h)

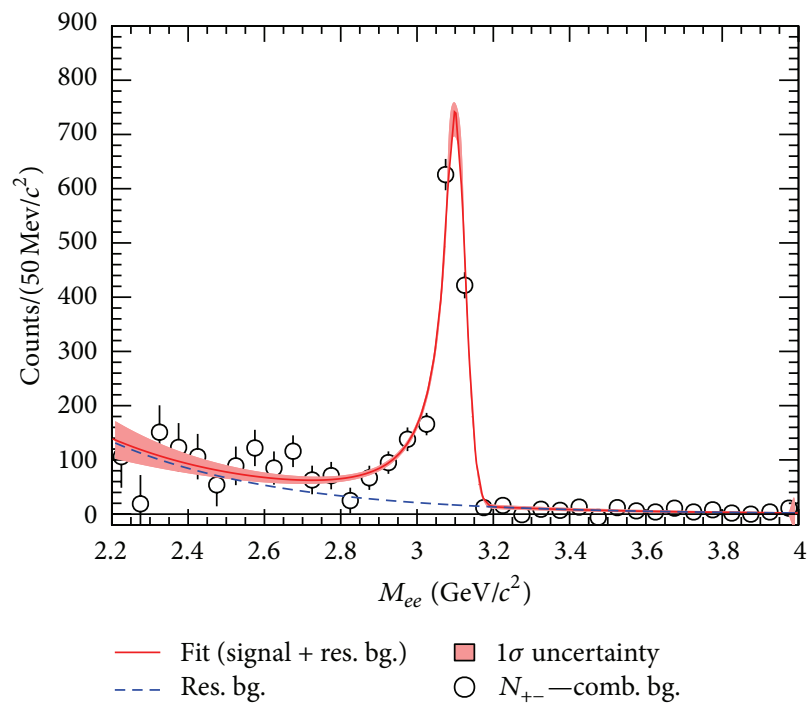

(j)

FIGURE 7: Dielectron mass spectra and $J / \psi$ signals for various hadron contamination levels.

TABLe 2: Photonic electron yield in the $m_{e^{+} e^{-}}<0.2 \mathrm{GeV} / c^{2}$ mass range for various hadron contamination levels. The statistical uncertainty is $0.05 \%$. The bias is defined as the relative difference between the observed yield and the signal in the pure electron sample.

\begin{tabular}{lcc}
\hline & Signal & Bias \\
\hline Electrons only & $4.18 \times 10^{6}$ & 0 \\
Electrons + 10\% hadrons & $4.18 \times 10^{6}$ & $0.1 \%$ \\
Electrons + 25\% hadrons & $4.20 \times 10^{6}$ & $0.4 \%$ \\
Electrons + 50\% hadrons & $4.25 \times 10^{6}$ & $1.7 \%$ \\
Electrons + 100\% hadrons & $4.45 \times 10^{6}$ & $6.6 \%$ \\
\hline
\end{tabular}

$\Delta=\left(S_{\text {hadron }}-S_{\text {ele }}\right) / S_{\text {ele }}$, where $S_{\text {hadron }}$ is the yield extracted with hadron contamination and $S_{\text {ele }}$ is the signal in the pure electron sample. Figure 9 shows $\Delta$ as a function of single electron $p_{T}$. The difference is the highest for the $p_{T}$ ranges where the $d E / d x$ band of electrons overlaps with those of the kaons and protons. The bias is significant $\left(50 \%\right.$ for $p_{T} \sim$ $0.9 \mathrm{GeV} / c$ and $20 \%$ for $p_{T} \sim 0.5 \mathrm{GeV} / c$ ) for the highest levels of hadron contamination. However, it is less than $4 \%$ for the $25 \%$ hadron misidentification level and negligible for $10 \%$ hadron contamination.

The bias will have a noticeable effect on the photonic yield calculation for the hadron misidentification levels of $25 \%$ or higher at $p_{T}<1 \mathrm{GeV} / c$, where the phonic reconstruction efficiency is low (20\% or less). However, the typical purity in the experiments is much better than that shown in Figure 4 for $25 \%$ hadron misidentification and this effect is thus still small. To reduce the bias, a narrower mass range for the photonic background reconstruction should be used, for 


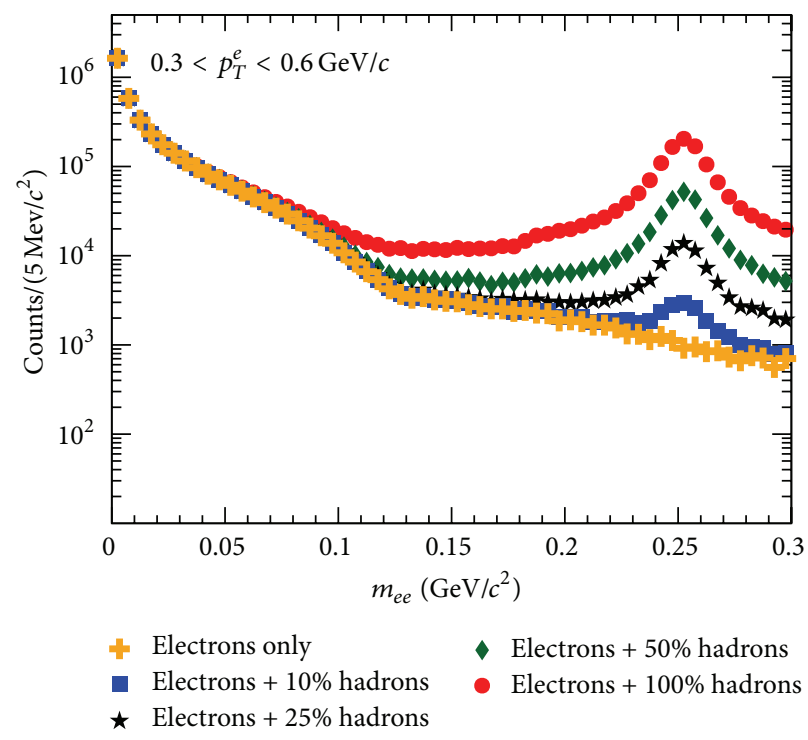

(a)

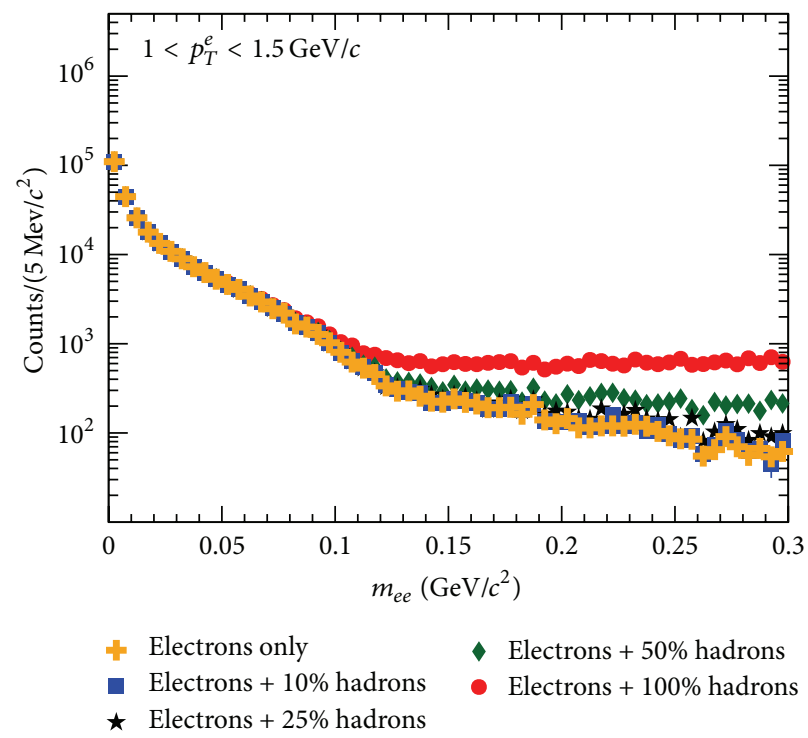

(c)

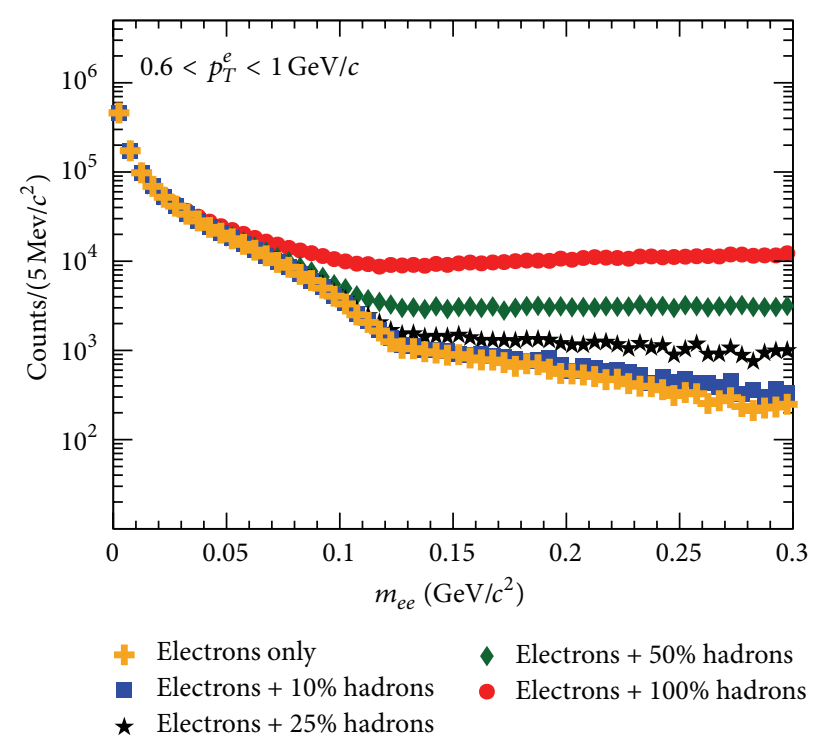

(b)

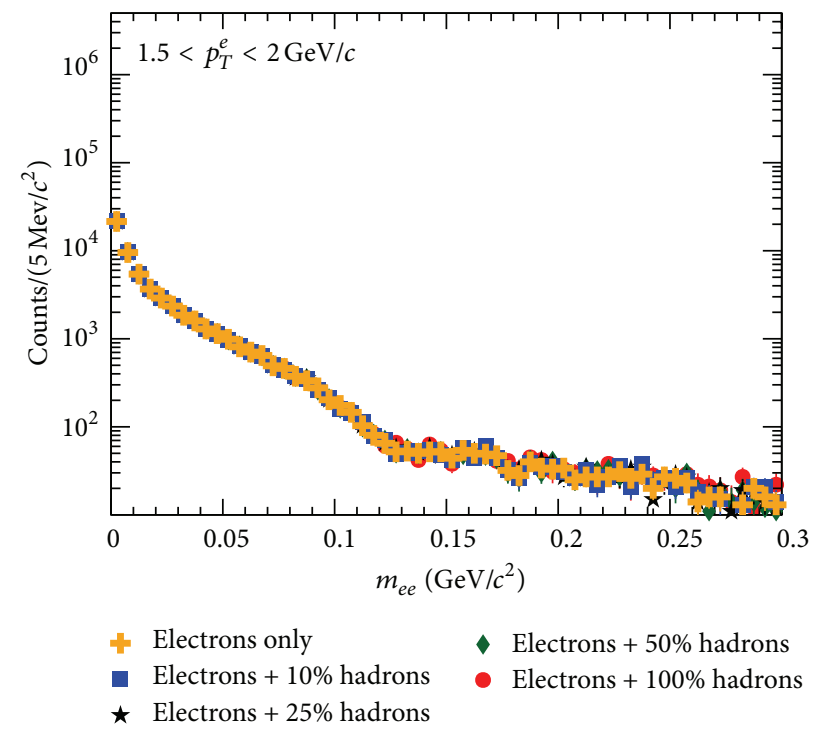

(d)

FIGURE 8: Dielectron mass spectra after combinatorial background subtraction for various hadron contamination levels. The peak around $m_{e^{+} e^{-}} \sim 0.26 \mathrm{GeV} / c^{2}$ is a $\phi \rightarrow K^{+} K^{-}$that has been reconstructed with the wrong assumption about the daughter mass.

example, $m_{e^{+} e^{-}}<0.15 \mathrm{GeV} / c^{2}$, or particle identification should be improved at low $p_{T}$ using a ToF detector.

The false signals from $\phi \rightarrow K^{+} K^{-}$and other hadronic decays (e.g., $K_{S}^{0} \rightarrow \pi^{+} \pi^{-}$) are important for the dielectron measurements $[26,27]$, where an excess yield in the $\rho$ mass region $\left(0.3-0.75 \mathrm{GeV} / c^{2}\right)$ is observed. They also affect the studies of direct virtual photon production [28], in which the virtual photons are identified through the low mass $e^{+} e^{-}$pairs $\left(m_{e^{+} e^{-}}<0.35 \mathrm{GeV} / c^{2}\right)$.

\section{Summary}

We investigated the effects of hadron misidentification on measurements of $J / \psi$ yield and on studies of the production of electrons from semileptonic heavy flavor hadron decays. When the misidentification level is high, there is a noticeable increase in the statistical uncertainty in the $J / \psi$ yield calculation. Overall, the extracted $J / \psi$ signal is consistent within the limits of statistical uncertainty with its real value and there is no significant bias. In the case of photonic background estimation in open heavy flavor measurements, bias is observed for high hadron contamination (where the extracted yield is higher than the real yield), although the effect is negligible for a hadron misidentification level of $10 \%$. Because the typical purity in the experimental studies is better than the purity assumed in this work, the effects of kaon, proton, and pion contamination on the heavy flavor measurements via semileptonic decays are thus negligible. 


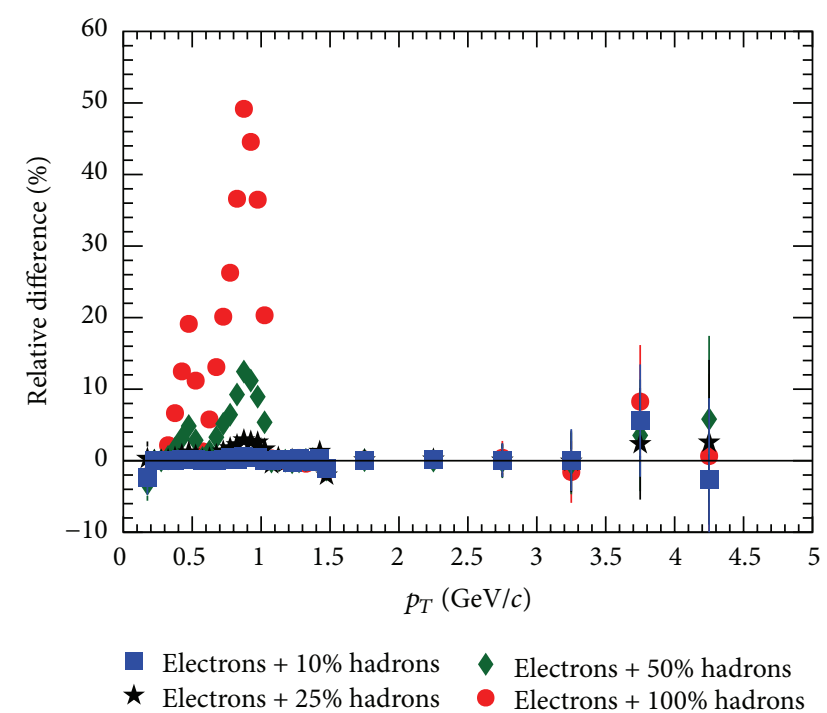

FIGURE 9: Relative difference $\Delta$ between observed yield and signal in a pure electron sample (bias due to hadron contamination) as a function of electron $p_{T}$ for various hadron misidentification levels.

\section{Conflict of Interests}

The author declares that there is no conflict of interests regarding the publication of this paper.

\section{Acknowledgment}

This work was supported in part by the Foundation for Polish Science Grant HOMING PLUS/2013-7/8.

\section{References}

[1] J. Adams, M. M. Aggarwal, Z. Ahammed et al., "Experimental and theoretical challenges in the search for the quark-gluon plasma: the STAR Collaboration's critical assessment of the evidence from RHIC collisions," Nuclear Physics A, vol. 757, no. 1-2, pp. 102-183, 2005.

[2] K. Adcox, S. S. Adler, S. Afanasiev et al., "Formation of dense partonic matter in relativistic nucleus-nucleus collisions at RHIC: experimental evaluation by the PHENIX Collaboration," Nuclear Physics A, vol. 757, no. 1-2, pp. 184-283, 2005.

[3] S. K. Das, V. Chandra, and J.-E. Alam, "Heavy-quark transport coefficients in a hot viscous quark-gluon plasma medium," Journal of Physics G: Nuclear and Particle Physics, vol. 41, no. 1, p. 015102, 2014.

[4] G. D. Moore and D. Teaney, "How much do heavy quarks thermalize in a heavy ion collision?" Physical Review C, vol. 71, no. 6, Article ID 064904, 19 pages, 2005.

[5] N. Brambilla, S. Eidelman, B. K. Heltsley et al., "Heavy quarkonium: progress, puzzles, and opportunities," The European Physical Journal C, vol. 71, article 1534, 2011.

[6] H. Agakishiev, M. M. Aggarwal, Z. Ahammed et al., "High $p_{T}$ nonphotonic electron production in $p+p$ collisions at $\sqrt{s}=$ 200 GeV," Physical Review D, vol. 83, Article ID 052006, 2011.

[7] B. Abelev, M. M. Aggarwal, Z. Ahammed et al., "Transverse momentum and centrality dependence of high- $p_{T}$ nonphotonic electron suppression in $\mathrm{Au}+\mathrm{Au}$ collisions at $\sqrt{s_{N N}}=200 \mathrm{GeV}$,' Physical Review Letters, vol. 98, no. 19, Article ID 192301, 6 pages, 2007.

[8] A. Adare, S. Afanasiev, C. Aidala et al., "Heavy-quark production in $p+p$ and energy loss and flow of heavy quarks in $\mathrm{Au}+\mathrm{Au}$ collisions at $\sqrt{s_{N N}}=200 \mathrm{GeV}$," Physical Review C, vol. 84, no. 4, Article ID 044905, 42 pages, 2011.

[9] E. P. de Oliveira Filho, "Measurements of the correlation between electrons from heavy-flavour hadron decays and light hadrons with ALICE at the LHC," Journal of Physics: Conference Series, vol. 509, Article ID 012060, 2014.

[10] P. Huck, "Di-electron production in $\mathrm{Au}+\mathrm{Au}$ collisions from 19.6 to $62 \mathrm{GeV}$ from STAR," in Proceedings of the Thermal Radiation Workshop, Brookhaven National Laboratory, Upton, NY, USA, December 2012, https://drupal.star.bnl.gov/STAR/ presentations/thermal-radiation-workshop-2012/patrick-huck.

[11] T. Sjostrand, S. Mrenna, and P. Z. Skands, "PYTHIA 6.4 physics and manual," Journal of High Energy Physics, vol. 2006, no. 5, article 26, 2006.

[12] K. Adcox, S. S. Adler, N. N. Ajitanand et al., "Measurement of single electrons and implications for charm production in $A u+$ Au collisions at $\sqrt{s_{N N}}=130 \mathrm{GeV}$," Physical Review Letters, vol. 88, Article ID 192303, 2002.

[13] M. Anderson, J. Berkovitz, W. Betts et al., "The STAR time projection chamber: a unique tool for studying high multiplicity events at RHIC," Nuclear Instruments and Methods in Physics Research Section A: Accelerators, Spectrometers, Detectors and Associated Equipment, vol. 499, pp. 659-678, 2003.

[14] K. Aamodt, N. Abel, U. Abeysekara et al., "Transverse momentum spectra of charged particles in proton-proton collisions at $\sqrt{s}=900 \mathrm{GeV}$ with ALICE at the LHC," Physics Letters B, vol. 693, no. 2, pp. 53-68, 2010.

[15] I. Antcheva, M. Ballintijn, B. Bellenot et al., "ROOT-A C++ framework for petabyte data storage, statistical analysis and visualization," Computer Physics Communications, vol. 180, pp. 2499-2512, 2009.

[16] B. I. Abelev, S. Afanasiev, C. Aidala et al., "J/ $\psi$ production versus transverse momentum and rapidity in $\mathrm{p}+\mathrm{p}$ collisions at $\sqrt{s}=$ 200 GeV," Physical Review Letters, vol. 98, Article ID 232002, 2007.

[17] J. Beringer, J.-F. Arguin, R. M. Barnett et al., "Review of particle physics," Physical Review D, vol. 86, Article ID 010001, 2012.

[18] L. Adamczyk, J. K. Adkins, G. Agakishiev et al., "J/ $\psi$ polarization in $\mathrm{p}+\mathrm{p}$ collisions at $\sqrt{s}=200 \mathrm{GeV}$ in STAR," Physics Letters $B$, vol. 739, pp. 180-188, 2014.

[19] G. J. Erthal, SLAC-R-255: charmonium spectroscopy from radiative decays of the $\mathrm{J} / \psi$ and $\psi^{\prime}$ [Ph.D. thesis], Stanford University, Stanford, Calif, USA, 1982.

[20] R. A. Lee, "Radiative decays of the psi prime to all-photon final states," Tech. Rep. SLAC-282, 1985.

[21] K. Oyama, "ALICE detector status and upgrade plans," EPJ Web of Conferences, vol. 49, Article ID 02002, 2013.

[22] L. Adamczyk, J. K. Adkins, G. Agakishiev et al., "Elliptic flow of non-photonic electrons in Au+Au collisions at $\sqrt{s_{N N}}=200$, 62.4 and $39 \mathrm{GeV}$," http://arxiv.org/abs/1405.6348.

[23] L. Adamczyk, J. K. Adkins, G. Agakishiev et al., " $J / \psi$ production at low $p_{T}$ in $\mathrm{Au}+\mathrm{Au}$ and $\mathrm{Cu}+\mathrm{Cu}$ collisions at $\sqrt{s_{N N}}=200 \mathrm{GeV}$ $\mathrm{GeV}$ with the STAR detector," Physical Review C, vol. 90, Article ID 024906, 2014.

[24] B. Abelev, J. Adam, D. Adamová et al., "Inclusive $J / \psi$ production in pp collisions at $\sqrt{s}=2.76 \mathrm{TeV}$," Physics Letters $B$, vol. 718, no. 2, pp. 295-306, 2012. 
[25] B. Abelev, J. Adam, D. Adamova et al., "Measurement of electrons from semileptonic heavy-flavor hadron decays in $p p$ collisions at $\sqrt{s}=7 \mathrm{TeV}$,' Physical Review D, vol. 86, no. 11, Article ID 112007, 26 pages, 2012.

[26] L. Adamczyk, J. K. Adkins, G. Agakishiev et al., "Dielectron mass spectra from $\mathrm{Au}+\mathrm{Au}$ collisions at $\sqrt{s_{N N}}=200 \mathrm{GeV}$," Physical Review Letters, vol. 113, Article ID 022301, 2014.

[27] A. Adare, S. Afanasiev, C. Aidala et al., "Detailed measurement of the $e^{+} e^{-}$pair continuum in $p+p$ and $\mathrm{Au}+\mathrm{Au}$ collisions at $\sqrt{s_{N N}}=200 \mathrm{GeV}$ and implications for direct photon production," Physical Review C, vol. 81, Article ID 034911, 2010.

[28] C. Yang, "Direct photon production in $\mathrm{Au}+\mathrm{Au}$ collisions at $\sqrt{s_{N N}}=200 \mathrm{GeV}$ at STAR," Nuclear Physics A, vol. 931, pp. 691$695,2014$. 

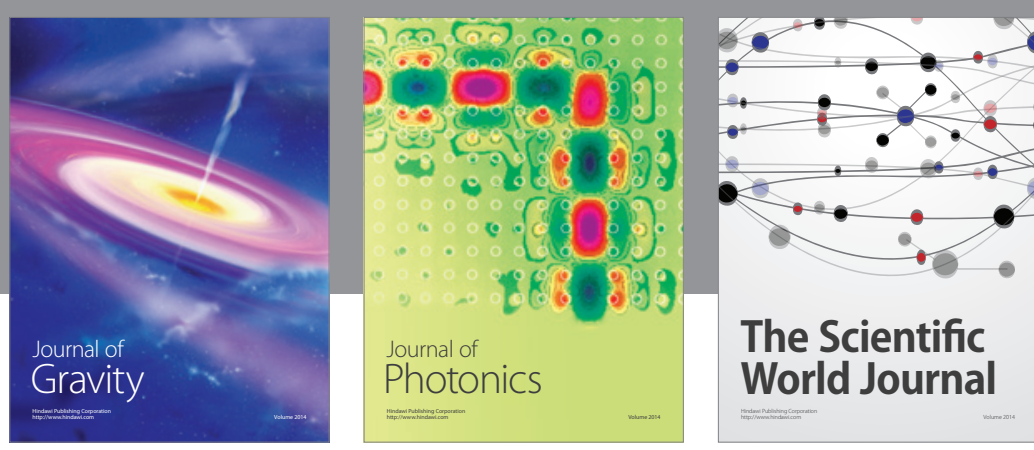

The Scientific World Journal
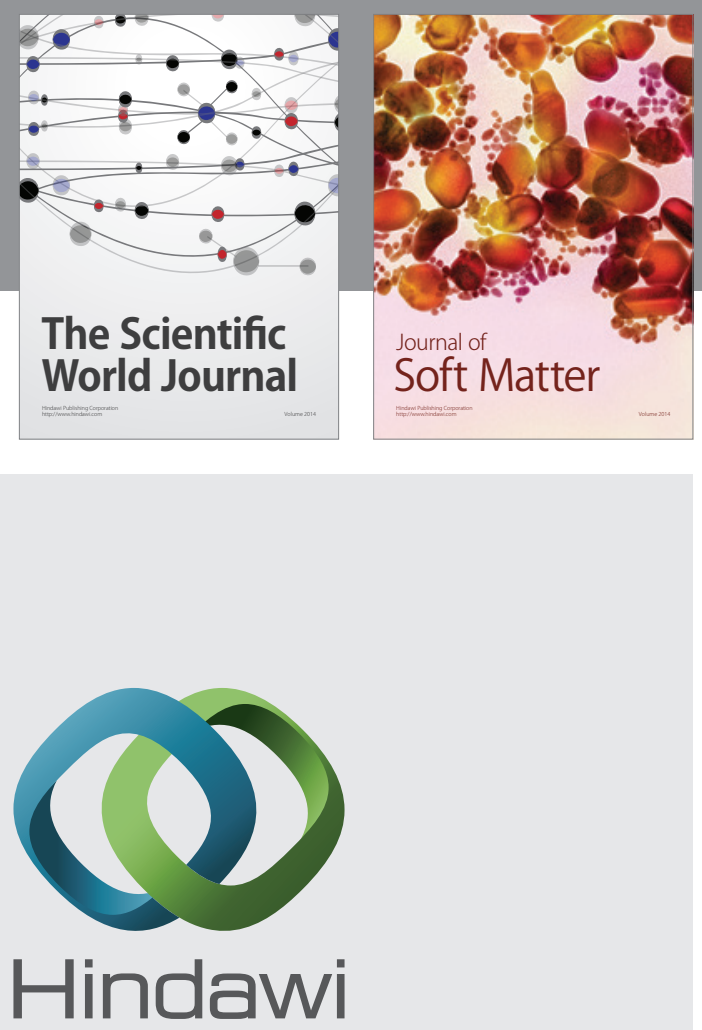

Submit your manuscripts at

http://www.hindawi.com

nternational Journal of

Statistical Mechanics
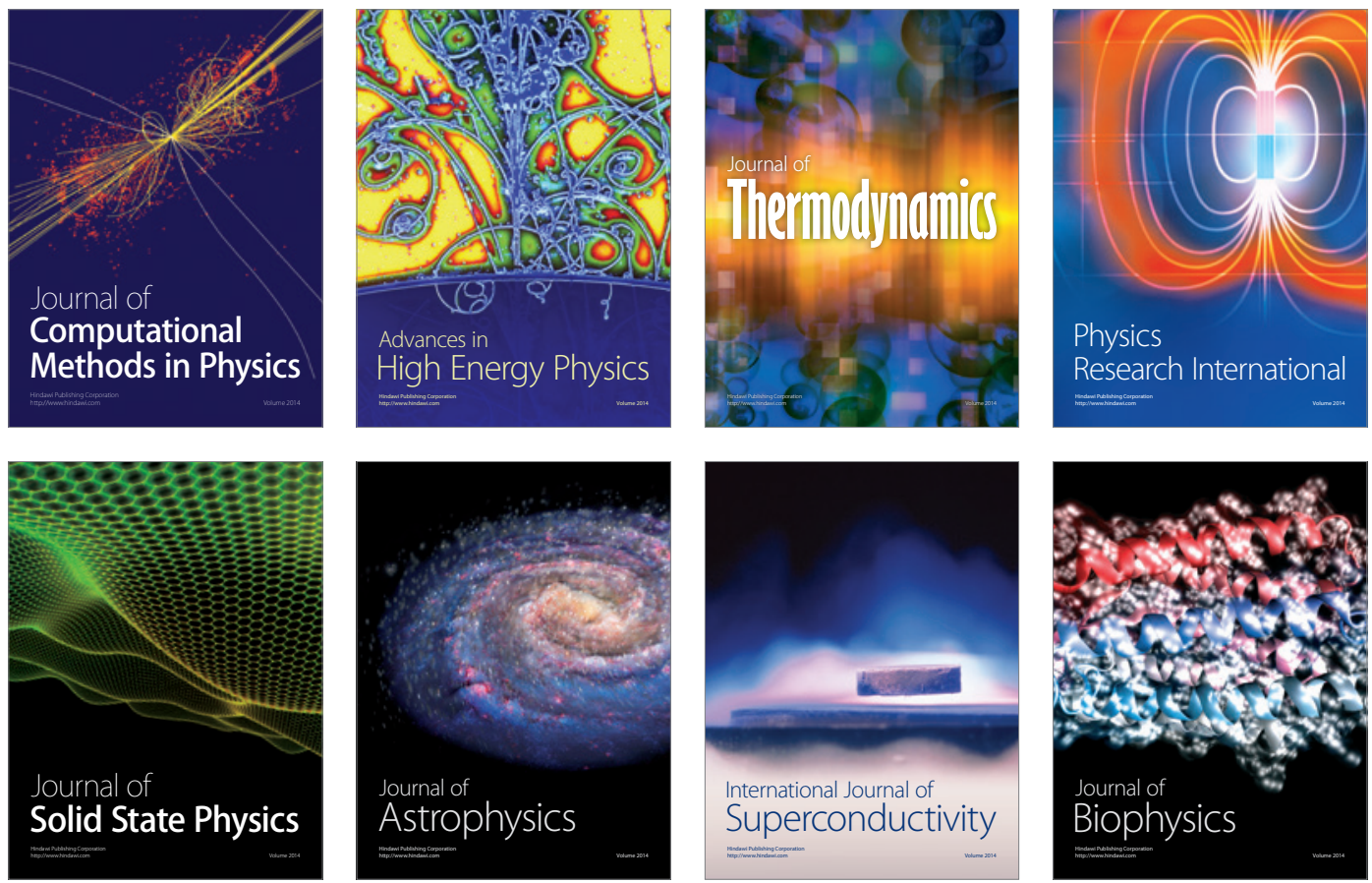
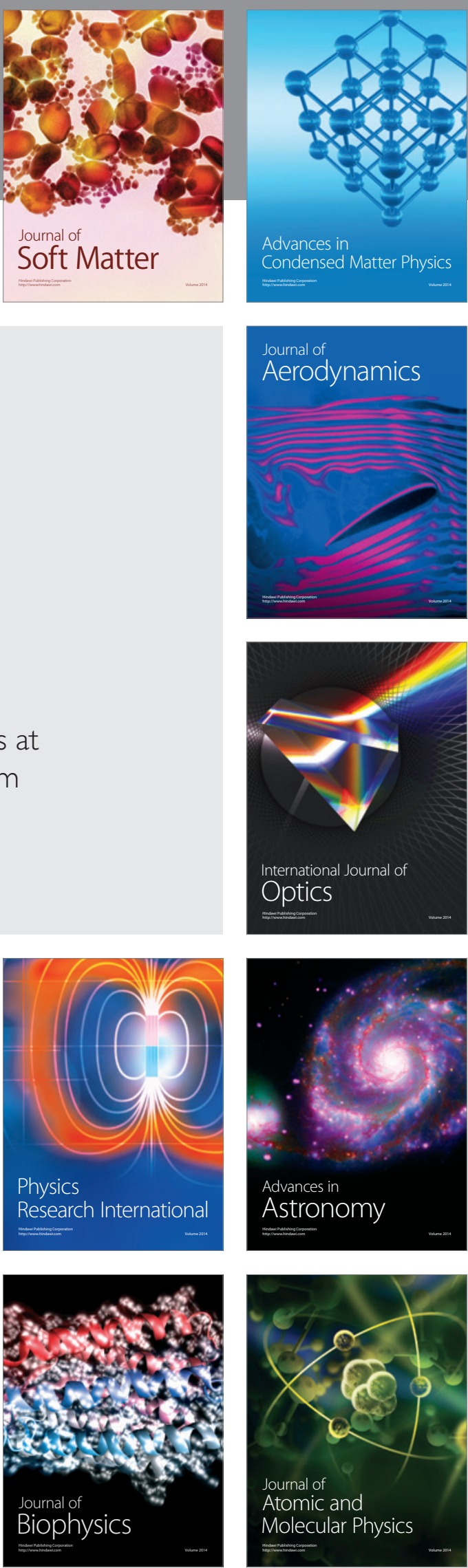